\title{
Learners' Aptitude towards Using Digital Techniques in Learning EFL: Internet Discourse
}

\author{
Bassam Almagarbeh (Corresponding author) \\ Al Karak Directorate of Education, Ministry of Education, Jordan \\ Email: bassam_magh@yahoo.com
}

Received: $16 / 11 / 2021$

Accepted: 09/02/2021

Published: 01/03/2022

Volume: 3 Issue: 2

How to cite this paper: Almagarbeh, B. (2022). Learners' Aptitude towards Using Digital Techniques in Learning EFL: Internet Discourse. Journal of Practical Studies in Education, $3(2), 1-6$

DOI: https://doi.org/10.46809/jpse.v3i2.42

This work is licensed under the Creative Commons Attribution International License (CC BY 4.0).http://creativecommons.org/licenses/by/4.0/

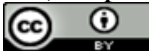

\begin{abstract}
The use of digital techniques has become very common inside the classroom as the past few decades witnessed a continuous development in the field of education. Concerning EFL language teaching and learning, digital information has developed to be usual practice in the classroom. The number of EFL learners who are considered "highly ready" for this kind of digital transformation is very limited. These EFL learners used personal devices such as laptops, IPads, smart devices with particular resource materials that were prepared before teaching and using these devices in the classroom. Generally, EFL teaching and learning appeared to be more exciting due to the use of smart devices and digital touch. They seemed to be creative and innovative inside the e-classroom. This paper aimed at investigating learners' aptitude towards the use of digital techniques in learning EFL. It attempted to examine the way digital learning media motivates EFL learning; then, sketches the difficulties experienced by EFL learners inside the e-classroom; and finally, recommends possible solutions to these difficulties. This research is designed to support professional learning and contribute to further investigations on similar themes. The study consisted of 30 EFL learners at the Institute of Languages at the University of Tabuk in Saudi Arabia selected randomly. It is based on Tubaishat and Lansari‘s (2011) Model of E-learning. Two research instruments were used; an Active Inspire Test and a questionnaire survey. The findings indicated that there are statistically significant differences at level $(0,05)$ in learners' aptitude towards the use of digital techniques in learning EFL inside the e-Classroom.
\end{abstract}

Keywords: Expectations, Experiences, Satisfaction, Distance learning, Online Learning Environment

\section{Introduction}

The In the conventional mode of learning, including EFL learning in Saudi Arabia, learners depend on classroom teachers as the only source of knowledge being passive listeners. That is, EFL learning was teacher-centered (Alenezi \& Shahi, 2015). This notion has changed during the digital transformation era: learners are the center of the learning process with the existence of e-classrooms. Learners with all their potentials, readiness, and contribution as the classroom composites support the learning process and possibly attempt to search knowledge on media or informal educational settings (Erizar, Andi \& Tuti, 2018). 
Nowadays, every EFL learner in Saudi Arabia is keen to be the center of the e-classroom and the monitor of his/her own encouraged by the digital transformation's concept. Every learner should be provided with the opportunity to be responsible for achieving designed learning objectives by being autonomous learners and included in the language learning process (Gibeault, 2018). The modifications over the process of learning concept are somewhat affected by the growth of human factors particularly during the digital transformation era in modern life. The development of e-classroom has invaded the learning process. The existence of digital techniques in the educational setting is exhibited by learning media, from simple to complicated forms as usually accessible in CALL centers and Language Labs (Rustam and Mengke, 2020). Digital techniques are there to facilitate the learning process.

This paper aims at examining learners' aptitude towards the use of digital techniques in EFL learning inside the eclassroom. It attempted to examine the way digital learning media motivates EFL learning then, sketches the difficulties experienced by EFL learners inside the e-classroom; and finally, recommends possible solutions to overcome difficulties.

\section{Theoretical Framework}

The process of computerizing learning and teaching relates to the early 1990s. Within almost thirty years of mobility, digital media and internet transformation are taking institutions and establishments to a new digital era and mobile IT processes (Zinder et al., 2016). Right from the 2000s until recently, digital media and internet platforms have been greatly affected by media transformation techniques and initiating innovative interaction channels with users. Digital communication supported greater expectations concerning multi-channel accessibility and transformed users' and services' experiences within an establishment and genuine-time communication method. Within the development of digital transformations, all sectors are carrying different initiatives to find out and take advantage of digital transformation benefits. This draws the attention of business operating models' transformation. Additionally, the administrative structures and procedures are to be restructured in a mode to control the complicated change. The innovative digital transformation-related business model necessitates a vast range of skills and capacities to be developed, beginning with the effect on individuals' mindset and culture of the organizations and the willingness to adapt (Mugge, 2017). All the components are related to each other into a phenomenon known as digital transformation, apparently posturing a general readiness for adaptation and still being a difficulty for industrialists.

\section{Definition of Digital Transformation}

Based on related literature, many definitions of digital transformation have been provided (Kokkinakos, Markaki, and Koussouris 2016). According to Mazzone (2014:p.5) digital transformation "is the deliberate and ongoing digital evolution of a company, business model, idea process, or methodology, both strategically and tactically”. Also, Fitzgerald et al. (2013:p.9) define digital transformation as the " use of new digital technologies, such as social media, mobile, analytics or embedded devices, to enable major business improvements like enhancing customer experience, streamlining operations or creating new business models ".

Therefore, the definition of digital transformation (DT, hereafter) is discussed from the perspective of three main characteristics that include; organizational (BMWi, 2015), technological (Martin, 2008), and social aspects (Lee, Lee, and Chou, 2017).

\section{Using Digital Learning Techniques in EFL Setting}

Using digital learning techniques is very beneficial in many ways (Tang, 2019). For instance, it helps in uniformly offering learning materials using an interesting process of learning. Also, digital learning is very beneficial for its capability to reduce and minimize learning time, develop learners' quality and build learners' dependency (Adnyani, Mahayanti \& Suprianti, 2020). Additionally, using technology in classroom teaching helps improve positive assertiveness towards the process of learning in an EFL setting. According to Rustam and Mengke (2020), applying digital tools in the process of learning widens learners' roles and contributions, as they do not need to refer to their teachers now and then. They just need to view oral discussion anywhere at any time. Thus, they have enough time to revisit recorded lessons and information when needed. Learners in their learning process should use digital techniques as learning aids, but actually, this point is often ignored due to certain factors, such as limited space and time inside the classroom, problems of internet connectivity, funding, financial support, etc.

However, none of these aspects is needed, since essentially several kinds of media will work according to definite settings and needs, such as space, time, fiscal support, and courses to be taught. A definite type of digital technology has specific features and the capacity in delivering knowledge (Sauro \& Sundmark, 2019). Learners should realize the features and capacity of every kind of digital technology so that they can choose which one best relates to the learning demands and conditions. Digital tools such as Audio or Audio-visual CDs, projectors, smart devices, laptops, IPads, etc. are accessible in the nowadays classroom and are found in the market. When the accessible audio resources are not appropriate, learners can refer to e-materials by themselves in a simple manner and correspondingly fewer efforts and low prices. Audiovisual digital 
techniques making for e-classroom application just necessities a recorder and a better speaker of English; that might be the monitor or learner him/herself.

\section{The creativity of Using Digital Tools in EFL e-Classroom}

Therefore, this is all about the concept of creativity in using digital tools in learning EFL inside the e-classroom. This is the most sophisticated concept that a learner should be convinced about that digital media would make their learning and life in the educational setting easier. In his practical experience of teaching EFL learners at the Institute of Languages at the University of Tabuk, the researcher, found that most of the learners have not revealed to show interest in using any kind of digital tools. They preferred conventional classes most often, sending off an impression of independent learning. That is to mention, nowadays' EFL classrooms are equipped with practices relating to deductive learning. Digital techniques are often viewed as accessible in the e-classroom. An active learner of English may download resourceful teaching materials. The internet is an unrestricted source of accessible and available materials. Using digital techniques in an EFL e-classroom is the wheel of change as a kind of learning modernization.

According to Montanero and Marques (2018), digital techniques can transform the learning process. Digital techniques enable skillful learners to achieve maximum knowledge. On the other hand, media attracts and retains skillful learners by making them more open and oriented to using digital tools in the process of learning. In addition, technology boosts efficacy and job choices in the future. The development of digital media facilitates English learners to always use themselves to be digitally literate and operate with media to advance the learning process, optionally if they are dedicated to professionalism (Shadiev, Hwang \& Liu, 2019).

\section{Digital Age and EFL Learners}

Learners as a significant component in the learning process cannot evade the effect of the digital age which has already generated technologies. The digital era has realized the modern dimensions to the learning process that are not observable in the present conventional educational system (Uziak, et.al, 2018). Learning in the $21^{\text {st }}$ century is very complicated than ever before as it involves different skills that should be learned by the learners. In addition, establishing a sophisticated process of learning, the digital age affects the learner's contribution. Learners are needed to play their contribution by keeping up with digital transformation's development. Therefore, the main contribution of learners is to accept developing connections between their interests, awareness, and the actual skills that are expected to be taught (Yezli et al., 2020).

It is the mission of learners to accept technology as an effective technique to develop their skills and knowledge. In addition, many EFL learners appear to be not aware of traditional learning media and keep up with digital transformation. In the e-classroom, through peer-learning and teaching of learners' programs, in which the learning participants were given constructive feedback on learning presentation, did not appear to show much attention to the feedback provided, revealing the lack of presence of learning media. However, digital media were already kept accessible in the e-classroom, such as laptops, projectors, LCDs; they were left rather the same as in the following day of peer learning. Remarkably, when requested if the use of digital techniques in the classroom would be helpful to learners, almost all learners' answers fell into the satisfactory side of the measure (Erizar \& Tuti, 2018). This reveals that there is no consistency between the learners' views and their aptitudes. This can show that they only imitated; they were not authentic in providing answers to the questionnaires. In addition, this may show that they had an insufficient computer or digital skills, and therefore, they were not self-reliant.

In his professional development, the researcher had some knowledge concerning the use of e-classroom. Once, he was very interested in using audio-visual to inspire learners' learning interest in a listening e-class. It developed to be that digital techniques such as video were an unusual medium to help EFL learners listen and communicate. Later, the researcher piloted a classroom action research comprising the use of digital learning materials to develop the EFL learners' language performance (Kelly-Irving, 2017). Last year, the researcher conducted another e-classroom action research on using digital techniques as reflective tools in EFL e-classroom. The research showed that the use of digital techniques could help in providing some educational implications for the learning of EFL skills at the higher education level. However, it cannot be denied that EFL learners who experience the use of modern learning techniques in the digital age have more awareness related to the use of technology more than their instructors who just realize it in recent times.

In fact, in learning in the digital era, learners are more informative than their teachers in adopting and using digital media and accessing knowledge in different fields. They are not needed by their teachers and course materials. They use their prevailing knowledge and originate their meanings. It has given them autonomy and not to rely much on their teachers which was the case of conventional teaching methods (Gibeault, 2018).

EFL learners possess effective and thoughtful contributions in this digital era. Nowadays, learners right at their early ages develop great learning habits through using digital media. Their perceptions of the world are very distinctive compared to that of adults, appreciations to extraordinary access to evidence, individuals, and thoughts over highly interactive digital media. Nowadays learners are the newest model of a human being. Viewing the world of learners is not observing backward at the past - it is observing the future. They are the promising future (Shadiev, Hwang \& Liu, 2019).

In the meantime, using digital techniques assist learners to perform their classroom tasks. For example, in the past, learners used to depend on traditional to learn EFL. Those learning techniques can be substituted by projectors, LCD, 
Laptops, smartphones, and related digital media that are operative and resourceful for delivering classes. Even though there are helpful effects of the technology development, difficulties may also ascend. The major one, according to past literature (Montanero and Marques, 2018), is that a classroom equipped with digitally literate learners is being trained by linearthinking, technologically hindered learners. Learners have been visible to these technologies or comparable ones early throughout their seminal years, while their instructors have just been acquainted with it only lately. As a result, the learners are now and then more skilled with the technology than the learners. Some educational institutes were equipped with digital techniques. However, it appears only a few learners use them in the e-classroom. The relevant problem is: are they infrequently provided with the opportunity to learn how to use these digital techniques, or do they not have an interest in the technical expertise? Response to either question shows that the learners are kept behind concerning digital technology, at least the ones appropriate to the e-classroom operation. If it is real, then, these English learners do not mainly benefit from that. For instance, in respect to learners' digital development, Zinder et al., (2016) discuss the value of a digital learning system established by the project for the teaching of EFL skills in a digital era. The findings are that it provides a successful way to digital learning and innovative language skills. So far, training on learning digital media for English learners has been very infrequently held, at least locally and regionally. The suggestion can then create adjacent collaboration with learners' teaching and digital development institutions close by.

\section{Research Objectives}

This study attempts to achieve the following research questions:

i. To examine the learners' aptitude towards using digital techniques in learning EFL at the Institute of Languages at the University of Tabuk.

ii. To investigate whether there are any effects on students' learning levels due to the use of digital techniques in EFL classrooms?

\section{Research Questions}

To achieve the objectives of the research, this study addresses the following research questions:

i. What is the learners' aptitude towards using digital techniques in learning EFL at the Institute of Languages at the University of Tabuk?

ii. Are there any effects on students' learning levels due to the use of digital techniques in EFL classrooms?

\section{Methods}

This quantitative study included 30 EFL learners (males) from the Institute of Languages (hereafter, IL) at the University of Tabuk in Saudi Arabia for the academic year 2021/2022 during the second semester of their study program. The students were doing their first introductory year in languages and translation. The participants were selected randomly.

Two groups were included in the study; control (15 participants) and experimental (15 participants) groups. The control group used the conventional learning method and the experimental group implemented the e-learning method/ digital media techniques.

The study was motivated by the fact that students were encouraged to use digital devices (blackboard, ZOOM, smart devices, IPads, and Laptops, virtual and online classes) in their EFL learning process. Participants belonged to the same educational background. They were homogenous in age, language, cultural context, and academic system.

Two research instruments were used are; an Active Inspire Test and a Questionnaire Survey. The Active Inspire Test involved 25 items validated by a panel of experts in the field of E-learning and EFL field. The test aimed at measuring the differences in the test scores for both groups. The questionnaire included 12 paragraphs distributed over 4 headings. The participants were encouraged to learn EFL using different digital media techniques. Then, the researcher conducted an efficiency test and statistical analyses for concluding findings from participants' responses. However, similarities and differences were concluded based on the responses of both groups. Then, having EFL learners learn through digital techniques settings, post-questionnaire was disseminated to them. Then, the researcher conducted statistical analyses and examined collected data using the SPSS program.

\section{Results and Discussion}

The findings of the study indicated that there are no statistically significant differences at $(0,05)$ level due to the use of digital techniques. In addition, the results of the t-tests showed that the means for the experimental group is 19.1 with a standard deviation of 1.91 whilst the means for the control group was 14.2 with a standard deviation of 5.19.

Moreover, the results indicated that the statistical variance for both the means of the experimental and control group is 4 and the variance for the standard deviations of both groups is 3.47 at level (0.05). T-test value was 3.09 at level (0.00) and the t-test periodic value was 2.04. This endorses that result to be in favor of the experimental group. The researcher rejects the 
null-hypothesis set and implies a substitute in which there is a statistical difference at 0.05 in the effects on students' learning levels due to the use of digital techniques in the EFL classroom.

To validate the second hypothesis which assumes that there are no statistical differences in learners' aptitude when using digital techniques in learning EFL at the Institute of Languages at the University of Tabuk at 0.05, the researcher established the means and standard deviations based on the analysis of the items in the survey. The results revealed that the value of the means for the answers of the survey regarding the experimental group is 1.6-2 and 2.62-1.39 for the control group with a standard deviation value of $0.95-0.52$. The experimental group standard deviation values stretched between $0.68-0$.

This showed that there is a statistical difference and effect for learners' aptitude in using digital techniques in eclassroom. In addition, t-test independent results revealed that there is an effect of learners' aptitude because the t. test value was 3.09 at level 0.00 . This approves the refusal of the null hypothesis and accepts the replacement, which reveals, "there is an effect with the statistical difference in developing the aptitudes of EFL learners in using digital technology and establishing e-class environment.

Related to the given data analysis, the results of the first study hypothesis displayed that there are statistical differences in efficiency tests of EFL learners' aptitudes in favor of experimental group learning EFL using digital techniques or eclassroom. This is a pointer that using digital media techniques helped in developing learners' aptitudes, particularly during COVID-19 improved EFL learners' aptitude as shown in experimental group EFL learners' at the Institute of Languages at the University of Tabuk. They were very comfortable and creative. This might refer to the effect of digital media that results in aptitude and readiness of EFL learners and recalling data. To be very particular, EFL materials are interesting, and using digital learning supports learners in sincerely relating to the material. It also supports them taking less time, labor, and space through using digital media.

To conclude, EFL learners are ready to develop their learning and practices, using digital media in an educational setting by learning and cooperating with peers. Learners who are skillful in using digital media for learning can share expertise with classmates in collaborative action research.

Based on the observation conducted during learners' training on using digital techniques in the classroom, it reveals that most learners do not have awareness about applicable digital media in the classroom. Few learners have satisfactory visions into the practice of learning since they possess the opportunity to join and contribute to relatively a lot of top-down or centralized learning conducted by the teachers. Therefore, it is sensible for both learners and teachers to contribute to this kind of learning environment. Teachers and learners can keenly participate in online-digital learning held by the local administration board or within university-based services.

\section{Conclusion}

Having discussed issues on using digital techniques by EFL learners, here are some optional solutions to the difficulties encountered by EFL learners in achieving technological developments in the e-classroom. The solutions are recommended taking into consideration the characteristic of empowerment rather than the help of the decision-makers, which refers to reliance and insecurity. In addition, the active involvement of EFL teachers' syndicates is strategic to develop EFL learners' learning skills and competency. Within the e-classroom, English learners can establish a genuine setting for their digital learning development. By doing this, learners are not only sharing thoughts, information, skills, and know-how amongst themselves but also recruit favored bases of up-dated knowledge, e.g. blackboards, ZOOM, accessible digital platforms, etc., and online instructors with their funding and desires.

Learners' aptitude towards using digital techniques in the learning process in different fields has revolutionized the use of e-classroom. EFL learners should warmly welcome digital transformation in the learning process and genuinely apply it as a technique to support their learning process, of course, if they are dedicated to professional learning and development. Hence, they should constantly improve their creativity by using digital media for learning innovations. Collaborative learning eclassroom action research is strategic to enable EFL learners in maintaining pace with the growth of nowadays-digital technology relevant to the EFL setting. With the collaborative practice and actions, EFL learners should not stay still until they are very late.

\section{References}

Adnyani, Luh Diah Surya \& Mahayanti, Surya \& Suprianti, G. A. P. (2020). PowToon-Based Video Media for Teaching English for Young Learners: An Example of Design and Development Research. Alenezi, A. M., \& Shahi, K. K. (2015). Interactive e-learning through Second Life with Blackboard Technology. Procedia - Social and Behavioral Sciences, 176, 891-897. DOI: https://doi.org/10.1016/j.sbspro.2015.01.555.

BMWi. (2015). Industrie 4.0 und Digitale Wirtschaft - Impulse für Wachstum, Beschäftigung und Innovation, Bundes Ministe-rium für Wirtschaft und Energie, Berlin.

Erizar, Andi .S and Tuti. H (2018). The Effectiveness of Use Multimedia in English Teaching at SMPN 1 Meulaboh. Advances in Social Science, Education, and Humanities Research (ASSEHR), volume 208. 1st International Conference on Social Sciences and Interdisciplinary Studies (ICSSIS 2018). 
Fitzgerald M., Kruschwitz N., Bonnet D., and Welch M. (2013). Embracing Digital Technology: A New Strategic Imperative, MIT Sloan Management Review, Research Report.

Gibeault, M. J. (2018). Organization of Materials and Accessing the Library in Blackboard: A Learner-centered Usability Study. The Journal of Academic Librarianship, 44(2), 190-195. DOI: https://doi.org/10.1016/j.acalib.2018.02.008.

Kelly-Irving, M., Soulier, A., Mobile, L., Bartley, M., Raynaud, J.-P., Panico, L., \& Blane, D. (2017). Vignettes as a tool for research and teaching in life course studies: Interdisciplinary approaches. Advances in Life Course Research, 32, 3541. DOI: https://doi.org/10.1016/j.alcr.2016.09.001.

Kokkinakos P., Markaki O., Koussouris S., Psarras J. (2016). Digital transformation: is public sector following the enterprise 2.0 paradigm?, [In:] Digital Transformation and Global Society, pp. 96-105, Springer International Publishing.

Lee M.X., Lee Y.C., Chou C.J., 2017, Essential implications of the digital transformation in industry 4.0, Journal of Scientific \& Industrial Research, vol. 76, pp. 465-467.

Martin A. (2008). Digital literacy and the "digital society", Digital Literacies Concepts Policies Practices, 30, pp. 151-176.

Mazzone D. (2014). Digital or Death: Digital Transformation - The Only Choice for Business to Survive Smash and Conquer (1st ed.), Smashbox Consulting Inc.

Montanero, M., \& Marques, M.-J. (2018). "Explain it on the blackboard". An analysis of the educational interaction in mirror assessment activities. Learning, Culture, and Social Interaction. DOI: https://doi.org/10.1016/j.lcsi.2018.07.003.

Mugge P., Gudergan G., (2017). The Gap Between the Practice and Theory of Digital Transformation, Whitepaper. The 50thHawaiian International Conference of System Science.

Rustam Shadiev and Mengke Yang (2020) .Review of Studies on Technology-Enhanced Language Learning and Teaching. Sustainability, MDPI Review.

Sauro, S.; Sundmark, B (2019). Critically examine the use of blog-based fanfiction in the advanced language classroom. ReCALL 2019, 31, 40-55.

Shadiev, R.; Hwang, W.Y.; Liu, T.Y (2019). A study of the use of wearable devices for healthy and enjoyable English as a foreign language learning in authentic contexts. J. Educ. Technol. Soc. 2018, 21, 217-231.

Tang, X (2019). The effects of task modality on L2 Chinese learners' pragmatic development: Computer-mediated written chat vs. face-to-face oral chat. The system, 80, 48-59.

Tubaishat, A., \& Lansari, A. (2011). Are Students Ready to Adopt E-Learning? A Preliminary E-readiness Study of a University in the Gulf Region. International Journal of Information and Communication Technology Research, 1(5), 210-215

Yezli, Saber \& Khan, Anas. (2020). COVID-19 social distancing in the Kingdom of Saudi Arabia: Bold measures in the face of political, economic, social and religious challenges. Travel Medicine and Infectious Disease. 101692.

Zinder E., Yunatova I., (2016). Synergy for digital transformation: a person's multiple roles and subject domains integration, [in:] Digital Transformation and Global Society: First International Conference, June 22-24, Springer 